TITLE:

\title{
HGF-induced serine 897 phosphorylation of EphA2 regulates epithelial morphogenesis of MDCK cells in 3D culture
}

\section{$\operatorname{AUTHOR}(S)$ :}

Harada, Kohei; Negishi, Manabu; Katoh, Hironori

\section{CITATION:}

Harada, Kohei ...[et al]. HGF-induced serine 897 phosphorylation of EphA2 regulates epithelial morphogenesis of MDCK cells in 3D culture. Journal of Cell Science 2015, 128(10): 1912-1921

\section{ISSUE DATE:}

2015-05-15

URL:

http://hdl.handle.net/2433/230207

\section{RIGHT:}

(c) 2015. Published by The Company of Biologists Ltd.; The full-text file will be made open to the public on 15 May 2016 in accordance with publisher's 'Terms and Conditions for Self-Archiving'. 


\title{
HGF-induced serine 897 phosphorylation of EphA2 regulates epithelial morphogenesis of MDCK cells in 3D culture
}

\author{
Kohei Harada, Manabu Negishi and Hironori Katoh*
}

\begin{abstract}
Expression of EphA2 is upregulated in various cancers that are derived from epithelial cells and correlates with the ability of a cancer cell to undergo migration and invasion. Here we have investigated the role of EphA2 in the epithelial morphogenesis of Madin-Darby canine kidney (MDCK) cells in three-dimensional culture. We show that EphA2 is phosphorylated on serine residue 897 through hepatocyte growth factor (HGF) stimulation using a phosphatidylinositol 3-kinase (PI3K)-Akt-dependent mechanism and that this phosphorylation is required for the formation of extensions, the first step of tubulogenesis, in MDCK cysts. By contrast, stimulation using the ligand ephrinA1 dephosphorylates EphA2 on serine residue 897 and suppresses the HGF-induced morphological change. Furthermore, activation of the small GTPase RhoG is involved in the HGF-induced formation of extensions downstream of EphA2. These observations suggest that a ligand-independent activity of EphA2 contributes to epithelial morphogenesis.
\end{abstract}

KEY WORDS: EphA2, Epithelial-mesenchymal transition, Rho family GTPases

\section{INTRODUCTION}

Ephrin (Eph)-family receptor tyrosine kinases play pivotal roles in regulating cell migration and proliferation, and contribute to many steps during development and in the maintenance of tissue homeostasis through interactions with their ligand ephrins. Ephreceptor-ephrin interactions induce the tyrosine kinase activity of Eph receptors, and previous studies have shown that dysregulation of Eph-ephrin interactions induces cancer progression (Pasquale, 2010; Boyd et al., 2014). Among Eph receptors, EphA2 is known as a therapeutic target in cancers, because EphA2 is overexpressed in a variety of human cancers and functions to promote cancer cell migration and invasion. By contrast, stimulation of EphA2 with ephrinA1 suppresses the malignant behavior of cancer cells, indicating that EphA2 can have opposing roles in cancer cells in a ligand-dependent and -independent manner. Recent studies suggest that the oncogenic effects of EphA2 are attributed to its phosphorylation on serine residue 897 (S897), which is mediated by Akt activity downstream of growth factor receptor activation (Miao, et al., 2009; Miao et al., 2015).

One of the most important families of downstream signaling effectors in the Eph-ephrin system is the Rho-family of small GTPases, which are well known as regulators of the actin cytoskeleton in diverse cellular functions. Like other small GTPases, Rho-family GTPases function as molecular switches by

Laboratory of Molecular Neurobiology, Graduate School of Biostudies, Kyoto University, Yoshidakonoe-cho, Sakyo-ku, Kyoto 606-8501, Japan.

*Author for correspondence (hirokato@pharm.kyoto-u.ac.jp)

Received 24 September 2014; Accepted 20 March 2015 cycling between a GDP-bound inactive form and a GTP-bound active form. Following their activation, they interact with specific effectors that lead to various biological functions. The activation of Rho family GTPases requires GDP-GTP exchange, which is catalyzed by various guanine nucleotide exchange factors (GEFs). The Dbl family is one of the major classes of RhoGEFs that contain tandem Dbl homology (DH) and pleckstrin homology (PH) domains, mediating the GDP-GTP exchange through the DH domain. The regulation of RhoGEFs in the Eph-ephrin system has been extensively characterized in the nervous system (Egea and Klein, 2007; Pitulescu and Adams, 2010), and it is known that ephexin1 specifically binds to EphA receptors and induces activation of Rho GTPases - including RhoA, Rac1 and Cdc42 to promote axon outgrowth. By contrast, ephrin stimulation induces tyrosine phosphorylation of ephexin1, which enhances its exchange activity toward RhoA, leading to axon retraction (Shamah et al., 2001; Sahin et al., 2005). Furthermore, Vav-family RhoGEFs play an important role in the regulation of ephrin-stimulated Ephreceptor endocytosis, which is required for growth cone collapse during axon guidance (Cowan et al., 2005). Other studies have also implicated RhoGEFs that link Eph receptors to Rho GTPase activation - including TIAM1, intersectins, kalirin and ephexin5 in the regulation of axon guidance and dendritic spine morphogenesis (Irie and Yamaguchi, 2002; Penzes et al., 2003; Tolias et al., 2007; Margolis et al., 2010).

EphA2 also regulates the activities of Rho-family small GTPases in various cell types. For example, EphA2 activates RhoA and destabilizes adherens junctions in response to ephrinA1 stimulation in mammary epithelial cells (Fang et al., 2008a). EphA2-dependent RhoA activation is also involved in cell migration and cell-cell repulsion in cancer cells (Fang et al., 2005; Parri et al., 2007; Batson et al., 2014). The ephrin-EphA2dependent RhoA activation is mediated, at least in part, by Vav2 (Batson et al., 2014). However, ephrinA1 stimulation leads to RhoA inactivation in Madin-Darby canine kidney (MDCK) cells through p190RhoGAP (Wakayama et al., 2011). By contrast, stimulation of EphA2 through ephrinA1 results in the recruitment of Vav-family RhoGEFs to EphA2 and in activation of Rac1 to induce vascular assembly and cell migration in endothelial cells (Brantley-Sieders et al., 2004; Hunter et al., 2006); it also promotes invasion in prostate cancer cells (Lin et al., 2012). The activation of Rac1 is also required for ephrinA1-stimulated EphA2 endocytosis (Zhuang et al., 2007; Boissier et al., 2013). We have previously identified ephexin4 (also known as ARHGEF16) as a GEF for small GTPase RhoG that interacts directly with EphA2 (Hiramoto-Yamaki et al., 2010). Phosphorylation of S897 on EphA2 promotes the recruitment of ephexin4 to EphA2 (Kawai et al., 2013; Akada et al., 2014), and the EphA2-ephexin4 complex contributes to EphA2mediated cell migration and resistance to anoikis through the activation of RhoG, independently of an interaction with ephrin (Hiramoto-Yamaki et al., 2010; Harada et al., 2011). Activated 
RhoG recruits the ELMO-DOCK180 or ELMO-DOCK4 complex to the plasma membrane and induces activation of Rac, which is necessary for the promotion of cancer cell migration and invasion (Katoh et al., 2006; Hiramoto et al., 2006). Activation of RhoG also suppresses anoikis through a phosphatidylinositol 3-kinase (PI3K)Akt signaling pathway (Yamaki et al., 2007). These findings provide a molecular link between phosphorylation of EphA2 on S897 and tumor progression.

Most studies have highlighted the roles of EphA2 in cancer cells; however, it is expressed in a wide range of epithelial tissues (Lindberg and Hunter, 1990). A previous study has reported that EphA2 is required for the development of mammary glands (Vaught et al., 2009), although the molecular mechanism of EphA2mediated epithelial morphogenesis is poorly understood. Here, we approach this issue by using three-dimensional (3D) cell culture of MDCK cells, which is useful for investigating the formation of epithelial structure. In this system, when MDCK cells are cultured in Matrigel containing extracellular matrix (ECM) proteins, they form cysts comprising a monolayer of polarized cells surrounding a central lumen. In addition, following treatment of MDCK cysts with hepatocyte growth factor (HGF), they initially undergo a partial epithelial-mesenchymal transition (EMT) and produce actinrich extensions from their basal membrane, the first step of tubulogenesis (O’Brien et al., 2002; Zegers et al., 2003). Our results demonstrate that phosphorylation of EphA2 on S897 is required for HGF-induced formation of extensions and that RhoG functions as a key downstream regulator of EphA2 in HGF signaling. In a previous study in which MDCK cells were cultured in collagen gel, ephrinA1 stimulation was characterized as a negative regulator of HGF-induced branching morphogenesis (Miao et al., 2003), although its molecular mechanism remains to be determined. In this study, we show that stimulation of EphA2 with ephrinA1 in MDCK cysts causes dephosphorylation of EphA2 on S897 and suppresses the formation of extensions that are induced by HGF.

\section{RESULTS \\ Knockdown of EphA2 suppresses HGF-induced formation of extensions in MDCK cysts}

First, to examine the localization of EphA2 in MDCK cysts, we performed immunofluorescence analyses. Cells that had been grown in Matrigel for 10 days were stained with phalloidin (to label F-actin and visualize cyst structure) an antibody against EphA2, and they were imaged by using confocal microscopy. EphA2 was localized mainly at the basal surface of cysts and weakly detected at cell-cell contacts (Fig. 1A). To determine whether EphA2 participates in the formation and maintenance of cysts, we transfected MDCK cells with short hairpin RNA (shRNA) against control luciferase (shControl) or canine EphA2 (shEphA2) and established the control and EphA2-depleted cell lines (Fig. 1B). These cells were grown in Matrigel for 10 days to form cysts and then stained for F-actin. We confirmed knockdown of EphA2 in MDCK cysts that had been grown in 3D culture for 10 days by using immunoblotting (Fig. 1C) and immunofluorescence analysis (Fig. 1D). EphA2-depleted cells developed into normal cysts with a single lumen, and no significant difference was found in the percentage of normal cysts between shEphA2-expressing cells and untransfected or shControl-expressing cells (Fig. 1E,F), indicating that EphA2 is dispensable for the formation and maintenance of cysts. Next, we investigated the effect of EphA2 knock down on tubulogenesis that had been induced though HGF stimulation. When MDCK cysts are treated with HGF for 48 hours, they produce one or more actin-rich extensions from their basal membrane
(O'Brien et al., 2002; Zegers et al., 2003; Fig. 1G, upper panels). We quantified the cysts with extensions after treatment with HGF and found a marked decrease in the percentage of cysts with extensions formed from EphA2-depleted cells compared to those formed from untransfected or control cells (Fig. 1G,H). Collectively, these results suggest that EphA2 is required for the formation of extensions in MDCK cysts in response to HGF stimulation.

\section{Phosphorylation of EphA2 on S897 is required for HGF- induced formation of extensions in MDCK cysts}

To investigate the mechanism of EphA2-mediated formation of extensions, we used human wild-type EphA2 (EphA2-WT), which was resistant to the shRNA against canine EphA2, and two human EphA2 mutants, EphA2-SA and EphA2-KM (Fig. 2A). EphA2-SA contains an alanine substitution at the S897 phosphorylation site (Miao et al., 2009; Kawai et al., 2013), and EphA2-KM has a mutation in the tyrosine kinase domain (K646M) that impairs its tyrosine kinase activity (Wang et al., 2002). We introduced these constructs into EphA2-depleted cells, and immunoblot analysis showed similar expression levels of EphA2 in these cell lines and the control cells when cultured in two dimensions (Fig. 2B) and grown in Matrigel for 10 days (Fig. 2C). Also, the phosphorylation status of EphA2 in these cell lines did not change (Fig. 2C). These cell lines were treated with HGF for 48 hours after formation of cysts. Expression of EphA2-WT or EphA2-KM restored HGFinduced formation of extensions, whereas expression of EphA2-SA failed to rescue the morphological defect caused by EphA2 knock down (Fig. 2D,E). Taken together, these results suggest that phosphorylation of S897 of EphA2, but not its tyrosine kinase activity, is required for HGF-induced formation of extensions in MDCK cysts.

\section{EphA2 is phosphorylated on S897 through HGF and dephosphorylated through ephrinA1 in MDCK cysts}

To determine whether HGF stimulation induces phosphorylation of EphA2 on S897 in MDCK cysts, lysates were prepared from cysts that had been treated with or without HGF and then analyzed by immunoblotting with an antibody against phosphorylated EphA2 at S897 (pS897-EphA2). Stimulation of MDCK cysts with HGF significantly enhanced the level of phosphorylation at S897 of EphA2 (Fig. 3A). EphA2 serves as a substrate of Akt downstream of growth factor receptors and promotes cell migration and invasion in glioma (Miao et al., 2009). Based on that study, we speculated that the activation of PI3K-Akt signaling is involved in the HGF-induced phosphorylation of EphA2 on S897 in MDCK cysts. We treated the cysts with LY294002 (a PI3K-specific inhibitor) or MK-2206 (an Akt-specific inhibitor) and found that both of these inhibitors completely suppressed HGF-induced phosphorylation of EphA2 on S897 in MDCK cysts (Fig. 3A). These results suggest that HGF induces phosphorylation at S897 of EphA2 through a PI3KAkt-dependent signaling pathway. LY294002 also suppressed the HGF-induced formation of extensions (Fig. 3B,C). Next, we examined the distribution of EphA2 that was phosphorylated at S897 in HGF-stimulated cysts by using immunofluorescence analysis with an antibody against pS897-EphA2, and pS897-EphA2 was observed to be localized in actin-rich areas at the tip of extensions (Fig. 3D).

A previous study has reported that ephrinAl stimulation suppresses HGF-induced cell protrusions in MDCK cells in collagen gel (Miao et al., 2003). In agreement with this, we observed that treatment with ephrinA1 significantly blocked the 
A

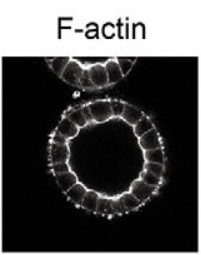

C

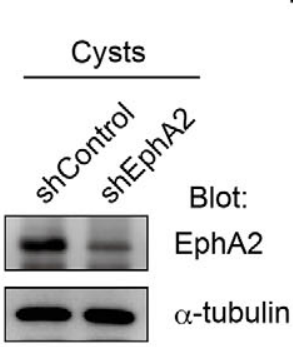

E

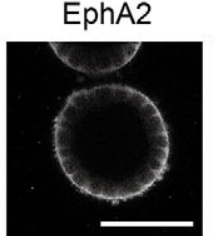

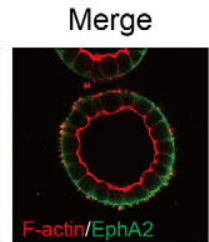

B

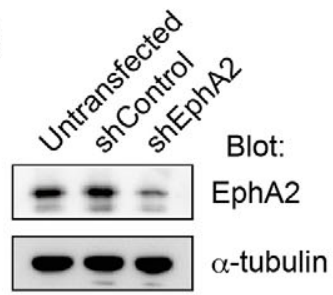

D

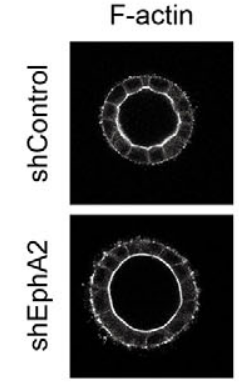

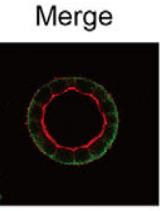

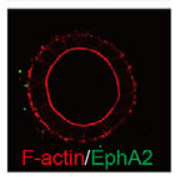

ns

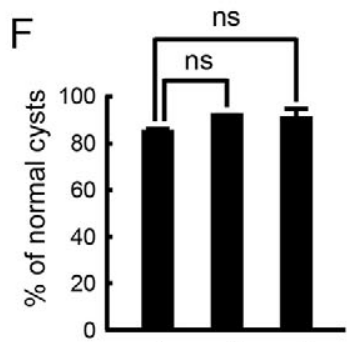

G

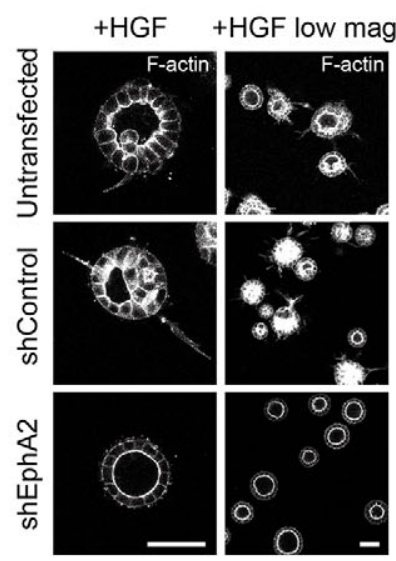

shEphA2
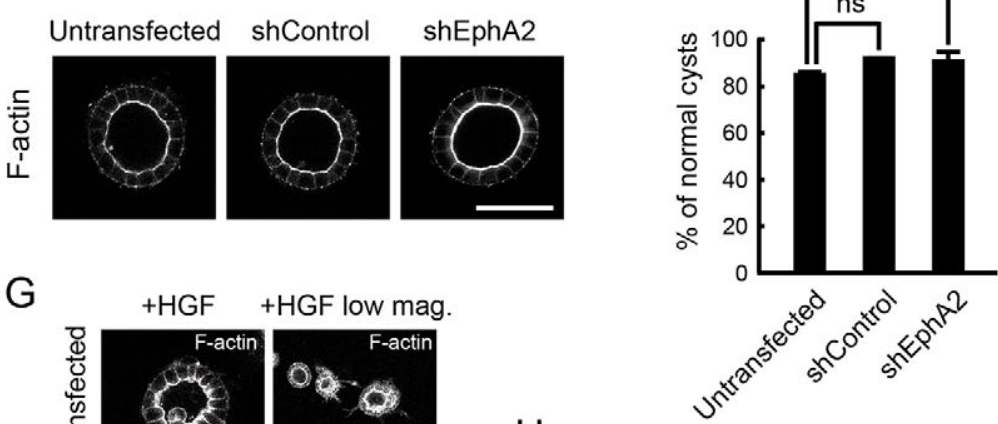

$\mathrm{H}$

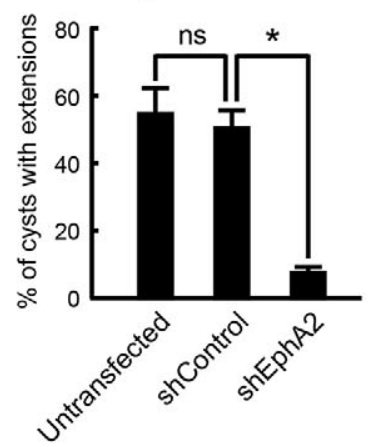

Fig. 1. Knockdown of EphA2 suppresses HGFinduced formation of extensions in MDCK cysts. (A) MDCK cells were grown in Matrigel for 10 days, then they were fixed and stained with an antibody against EphA2 antibody (green) and phalloidin (red, labeling for F-actin to visualize cyst structure). (B) Cell lysates from MDCK cells expressing a control shRNA against luciferase (shControl) or an shRNA against EphA2 (shEphA2) were analyzed by immunoblotting with antibodies against EphA2 and $\alpha$-tubulin. (C) MDCK cells were grown in Matrigel for 10 days, and the cyst lysates were analyzed by immunoblotting with antibodies against EphA2 and $\alpha$-tubulin. (D) MDCK cells expressing shControl or shEphA2 were grown in Matrigel for 10 days and then fixed. Cysts were stained with an antibody against EphA2 (green) and phalloidin (red). (E) MDCK cysts were stained with phalloidin. (F) Quantification of the percentage of normal cysts. Data are the means \pm s.e.m. from three independent experiments (ns, not significant; one-way ANOVA with Dunnett T3). (G) MDCK cells expressing shControl or shEphA2 were grown in Matrigel for 8 days, followed by treatment with $50 \mathrm{ng} / \mathrm{ml} \mathrm{HGF}$ for 48 hours. Cysts were stained with phalloidin. Low mag., low magnification. $(H)$ Quantification of cysts with one or more actin-rich extensions. Data are the means \pm s.e.m. of three independent experiments $\left({ }^{*} P<0.05\right.$; ns, not significant; one-way ANOVA with Dunnett T3). At least 100 cysts were counted for each experiment. Scale bars: $50 \mu \mathrm{m}$. formation of extensions upon stimulation with HGF (Fig. 4A,B). To determine whether ephrinA1 stimulation alters the level of phosphorylation of EphA2 at S897, we analyzed lysates of cysts that had been co-treated with HGF and ephrinA1 by immunoblotting with antibodies against EphA2 and pS897-EphA2. Treatment with ephrinA1 abolished HGF-induced upregulation of pS897EphA2 (Fig. 4C). Collectively, these results suggest that ephrinA1 negatively regulates HGF-induced formation of extensions in MDCK cysts through dephosphorylation of EphA2 at S897. We also examined phosphorylation of EphA2 at tyrosine residue 588 (Y588) in MDCK cysts. Y588 is located in the juxtamembrane region of EphA2, and phosphorylation of this tyrosine residue is required for tyrosine kinase activation of Eph receptors by ligand ephrins (Kullander and Klein, 2002; Fang et al., 2008b). Although stimulation with ephrinA1 enhanced the level of phosphorylation of EphA2 at Y588, stimulation with HGF had no effect on the phosphorylation of endogenous EphA2 (Fig. 4C) or EphA2-WT and EphA2-KM at Y588 in EphA2-depleted MDCK cysts (Fig. 4D). These results further support our conclusion that the tyrosine kinase activity of EphA2 is dispensable for the HGF-induced formation of extensions in MDCK cysts.

\section{Ephexin4 mediates HGF-induced formation of extensions through the activation of RhoG}

We have previously revealed that phosphorylation of EphA2 at S897 regulates the interaction with ephexin4 and RhoG activation (Hiramoto-Yamaki et al., 2010; Kawai et al., 2013). To determine whether ephexin4 is a candidate downstream effector of EphA2 in HGF-stimulated MDCK cysts, we established an MDCK cell line that expressed shRNA against canine ephexin4 (shEphexin4), which effectively reduced the amount of endogenous ephexin4 in MDCK cysts (Fig. 5A). Depletion of ephexin4 did not noticeably 
A
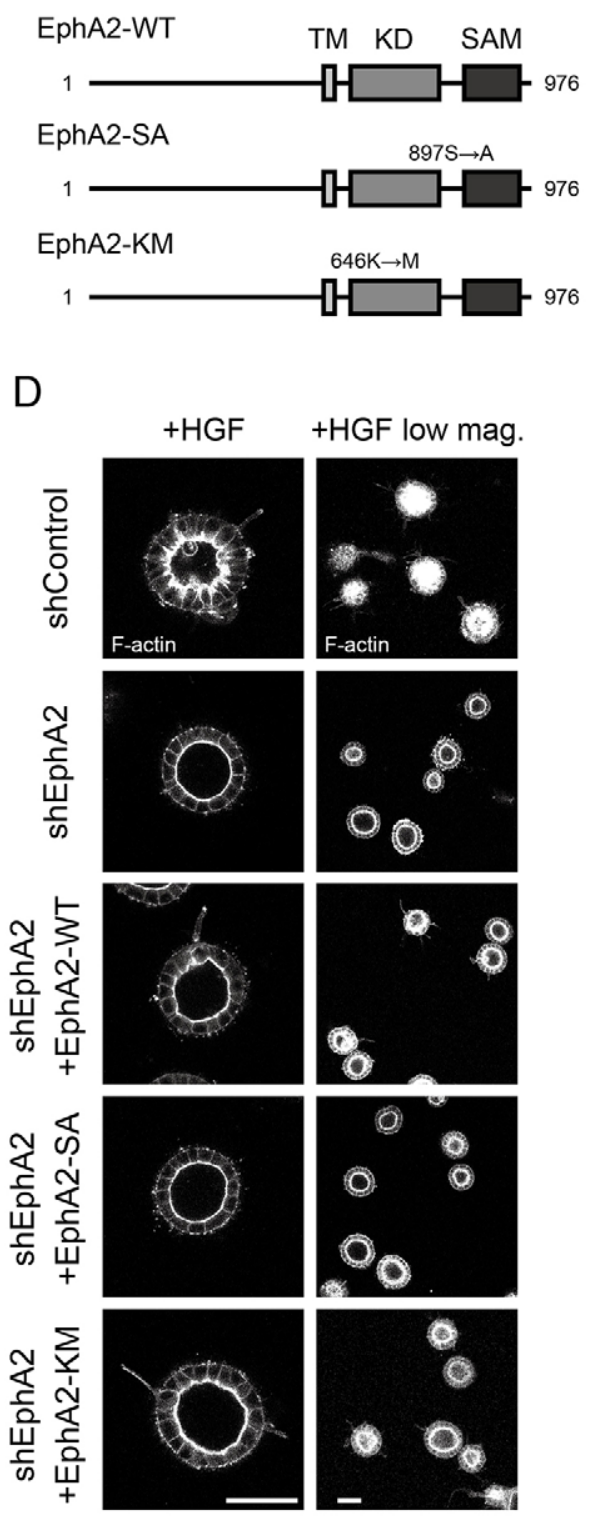

B

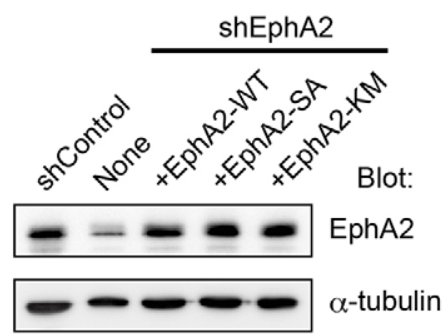

C

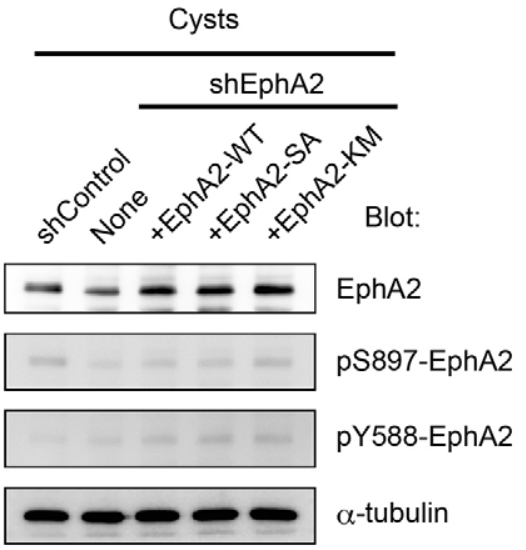

E

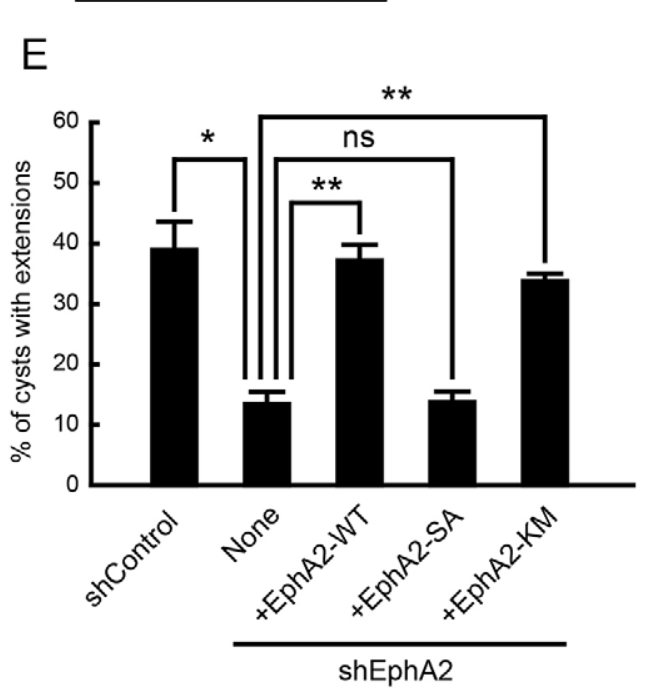

Fig. 2. Phosphorylation of EphA2 on $\mathbf{S 8 9 7}$ is required for HGF-induced formation of extensions in MDCK cysts. (A) The EphA2 constructs used in this study. TM,

transmembrane; KD, kinase domain; SAM, sterile- $\alpha$-motif. Numbers indicate amino acid position within the sequence. (B) Lysates from MDCK cells expressing the indicated shRNAs or co-expressing the EphA2 constructs (-WT, -SA or -KM) were analyzed by immunoblotting with antibodies against EphA2 and $\alpha$-tubulin. (C) MDCK cells were grown in Matrigel for 10 days, and the cyst lysates were analyzed by immunoblotting with antibodies against EphA2, EphA2 phosphorylated at S897 (pS897-EphA2) or at Y588 (pY588-EphA2) and $\alpha$-tubulin. (D) MDCK cysts expressing the indicated plasmids were treated with HGF for 48 hours and then fixed. Cysts were stained with phalloidin. Low mag., low magnification. (E) Quantification of cysts with one or more actin-rich extensions. Data are the means \pm s.e.m. of four independent experiments $\left({ }^{*} P<0.05\right.$; ${ }^{* *} P<0.01$; ns, not significant; one-way ANOVA with Dunnett T3), and at least 100 cysts were counted for each experiment. Scale bars: $50 \mu \mathrm{m}$. affect the ability to form proper cyst structure (Fig. 5B, left panels; Fig. 5C). Next, we investigated the effect of knocking down ephexin4 on HGF-induced epithelial morphogenesis in MDCK cysts and observed a significant reduction in the percentage of cysts with extensions in ephexin4-depleted cysts relative to that in the control cysts (Fig. 5B, middle and right panels; Fig. 5D). We performed rescue experiments with Flag-tagged wild-type human ephexin4 (Flag-Ephexin4-WT) and an ephexin4 mutant containing E291A and N466A substitutions [Flag-Ephexin4-AA, analogous residues are required for the exchange activity of the RhoG activator SGEF (Ellerbroek et al., 2004; Fig. 5E)]. The RhoG activity in HEK293T cells that had been transfected with Flag-Ephexin4-WT or Flag-Ephexin4-AA was measured by using a pulldown assay with the GST-fused N-terminal RhoG-binding region of ELMO2 (GST-ELMO-NT), which could specifically interact with the active form of RhoG (Katoh and Negishi, 2003;
Hiramoto-Yamaki et al., 2010), and Ephexin4-AA failed to activate RhoG (supplementary material Fig. S1). In MDCK cysts, the expression of Flag-Ephexin4-WT completely rescued the defect in the formation of extensions caused by knock down of ephexin4, whereas expression of Flag-Ephexin4-AA did not (Fig. 5F,G). Taken together, these results suggest that ephexin4mediated RhoG activation is required for the HGF-induced formation of extensions.

\section{EphA2 mediates the activation of RhoG downstream of HGF signaling}

To confirm that HGF induces RhoG activation downstream of EphA2, we measured RhoG activity in the control and EphA2depleted MDCK cells. In the control cells, stimulation with HGF significantly enhanced the level of active RhoG, whereas it had little effect on the level of active RhoG in EphA2-depleted cells 


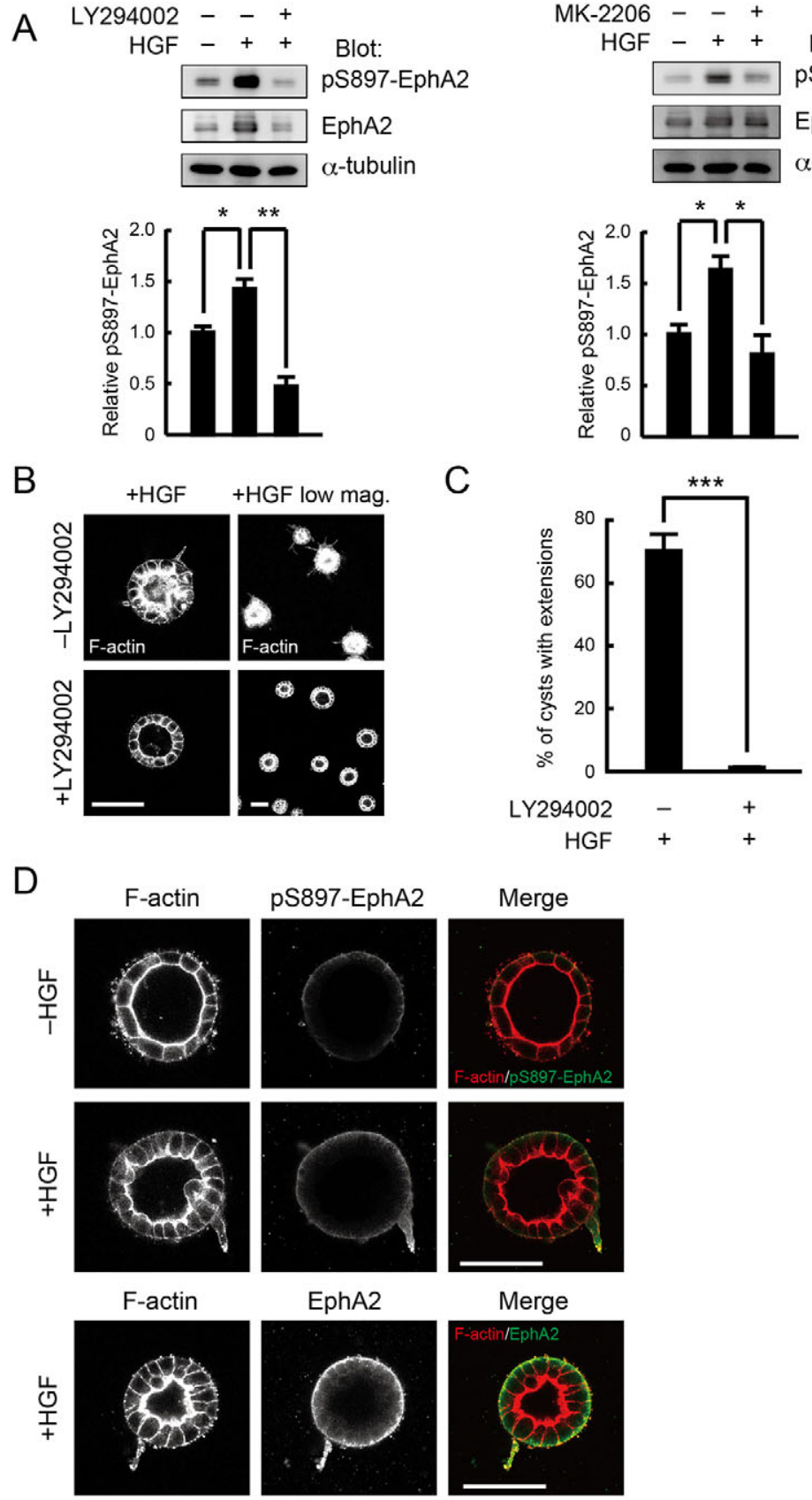

Fig. 3. EphA2 is phosphorylated on $\mathrm{S} 897$ through HGF in MDCK cysts. (A) MDCK cells were grown in Matrigel for 8 days and then treated with HGF for 48 hours in the presence or the absence of $20 \mu \mathrm{M}$ LY294002 or $1 \mu \mathrm{M}$ MK2206. The cyst lysates were analyzed by immunoblotting with antibodies against EphA2, pS897-EphA2 and $\alpha$-tubulin. The relative amount of pS897-EphA2 was determined by the amount of pS897-EphA2 normalized to the amount of total EphA2 in cyst lysates, analyzed by using Image J software. Data are presented as the means \pm s.e.m. from four independent experiments $\left({ }^{*} P<0.05 ;{ }^{* *} P<0.01\right.$; one-way ANOVA with Dunnett T3). (B) MDCK cells were grown in Matrigel for 8 days, followed by treatment with HGF for 48 hour in the presence and the absence of $20 \mu \mathrm{M}$ LY294002. Low mag., low magnification. (C) Quantification of cysts with one or more actin-rich extensions. Data are the means \pm s.e.m. of four independent experiments ${ }^{* * *} P<0.001$; Student's $t$-test), and at least 100 cysts were counted for each experiment. (D) Following treatment with HGF for 48 hours, MDCK cysts were stained with phalloidin (red) and antibodies against pS897-EphA2 or EphA2 (green). Scale bars: $50 \mu \mathrm{m}$.
(Fig. 6A). Expression of EphA2-WT in EphA2-depleted cells restored the activation of RhoG upon stimulation with HGF, whereas expression of EphA2-SA did not (Fig. 6B). These results suggest that EphA2 mediates HGF-induced RhoG activation through phosphorylation at S897.

Next, we examined whether the expression of a constitutively active form of RhoG (RhoG-V12) could override the effect of EphA2 knock down on HGF-induced formation of extensions. We introduced green fluorescent protein (GFP)-tagged RhoG-V12 into EphA2-depleted cells, grew the cells in Matrigel and treated them with HGF for 48 hours. Although expression of RhoG-V12 had little effect on the formation of cysts, it could restore HGF-induced formation of extensions from EphA2-depleted cells (Fig. 6C,D), suggesting that RhoG activation is required downstream of EphA2 in HGF-induced formation of extensions.

\section{DISCUSSION}

EphA2, a member of the Eph-family receptor tyrosine kinases, is widely expressed in epithelial cells, but its functional roles and the molecular mechanisms remain incompletely understood. In this study, we show that EphA2 is required for the formation of extensions, the first step of HGF-induced remodeling into tubules in MDCK cysts. In addition, phosphorylation of EphA2 on S897 induced by HGF through PI3K and Akt is essential for this morphological change. Indeed, the PI3K inhibitor LY294002 inhibits the formation of extensions. By contrast, stimulation with ephrinA1 dephosphorylates EphA2 on S897 and negatively regulates HGF-induced formation of extensions. EphA2 is known to be a substrate of Akt (Miao et al., 2009; Kawai et al., 2013), and pS897-EphA2 and the products of PI3K, which activate Akt, show a similar localization at the leading edge of cellular extensions in the 
A

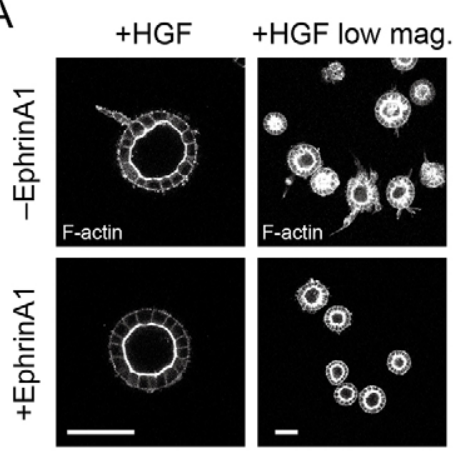

C

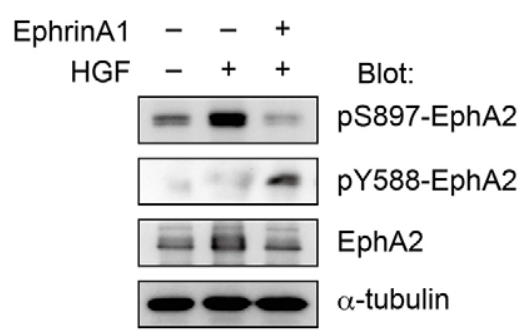

B

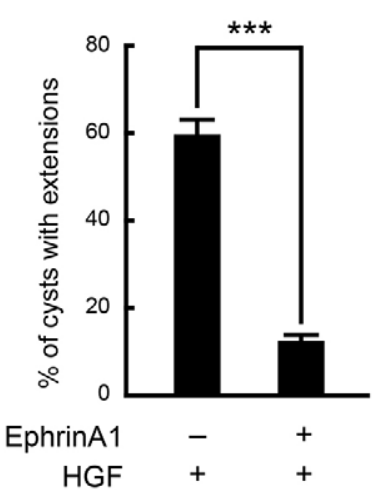

D

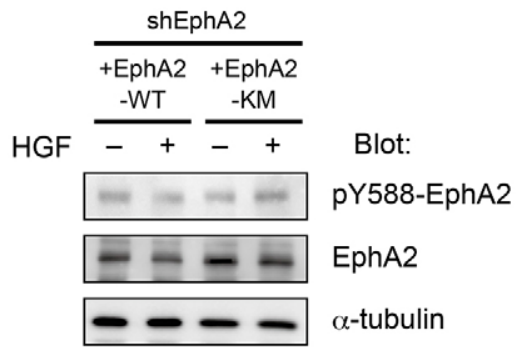

Fig. 4. EphrinA1 dephosphorylates EphA2 on S897 and suppresses extension formation in MDCK cysts. (A) MDCK cells grown in Matrigel were treated with $1 \mu \mathrm{g} / \mathrm{ml}$ ephrinA 1 for 24 hours in the presence of $\mathrm{HGF}$ and then stained with phalloidin. Low mag., low magnification. Scale bars: $50 \mu \mathrm{m}$.

(B) Quantification of cysts with one or more actin-rich extensions. Data are the means \pm s.e.m. of three independent experiments ( ${ }^{* * *} P<0.001$; Student's $t$-test), and at least 100 cysts in randomly selected fields were counted in one experiment. (C) MDCK cysts were treated with $1 \mu \mathrm{g} / \mathrm{ml}$ ephrinA 1 for 24 hours in the presence of HGF. The cyst lysates were analyzed by immunoblotting with antibodies against EphA2, EphA2 phosphorylated at S897 (pS897EphA2) or at Y588 (pY588-EphA2) and $\alpha$-tubulin. The relative levels of pS897-EphA2 and pY588-EphA2 were determined by the amount of pS897-EphA2 or pY588-EphA2 normalized to the amount of total EphA2 in cyst lysates, as analyzed by using ImageJ software. Data are presented as the means \pm s.e.m. from four independent experiments $\left({ }^{*} P<0.05\right.$; ns, not significant; one-way ANOVA with Dunnett T3).

(D) MDCK cells expressing the indicated plasmids were grown in Matrigel for 8 days and then treated with HGF for 48 hours. The cyst lysates were analyzed by immunoblotting with antibodies against EphA2, pY588-EphA2 and $\alpha$-tubulin.

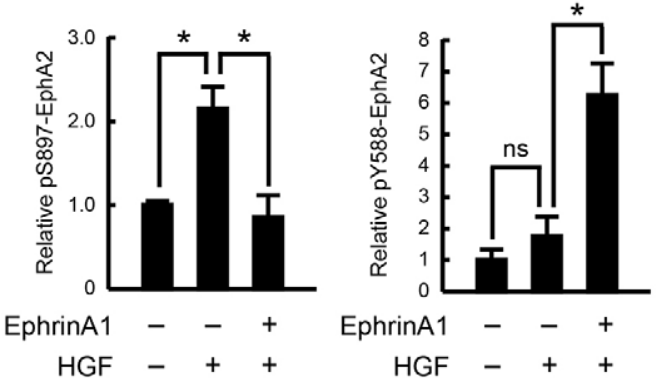

cysts (Yu et al., 2003), supporting the idea that HGF stimulation induces the activation of the PI3K-Akt signaling pathway followed by Akt-mediated phosphorylation of EphA2 on S897, leading to the formation of extensions in MDCK cysts. We also found that ephexin4, which is recruited to pS897-EphA2 (Kawai et al., 2013), serves as a RhoG activator and mediates HGF-induced formation of extensions. By contrast, EphA2 and ephexin 4 are not involved in the formation and maintenance of MDCK cysts. Taken together, our results suggest that the EphA2 and ephexin4 signaling pathway is dispensable for the acquisition of apico-basal polarity but that it plays an important role in the formation of extensions, an initial step that is required for the formation of tubular structures during epithelial morphogenesis. However, we cannot rule out the possibility that ephexin4 regulates the HGF-induced morphological change in parallel with EphA2 signaling. We tried to examine the localization of ephexin 4 in MDCK cysts, but we could not obtain clear images of the location of ephexin4. The reason for this is probably that our antibody against ephexin 4 was not suitable for immunofluorescence staining of the endogenous protein (HiramotoYamaki et al., 2010). We show here that the expression of a constitutively active form of RhoG can restore HGF-induced formation of extensions from EphA2-depleted cells. In addition, phosphorylation of EphA2 at S897 is required for HGF-induced RhoG activation. Therefore, these findings indicate that RhoG activation is required for HGF-induced formation of extensions downstream of EphA2 in MDCK cells. However, it is currently unknown whether phosphorylation of EphA2 at S897 alone is sufficient to produce actin-rich extensions in MDCK cysts independently of HGF. The first paper concerning phosphorylation of EphA2 at S897 has reported that a phosphomimetic mutation (S897D) of EphA2 has the same effects as an S897A mutation. Both S897A and S897D mutations completely abolish the promotion of cell migration by EphA2 (Miao et al., 2009). Therefore, it is possible that S897-phosphomimetic mutants of EphA2 also fail to act as EphA2 that is phosphorylated at S897 in MDCK cysts. In this study, we show that expression of constitutively active RhoG alone failed to produce actin-rich extensions in the absence of HGF. Thus, our results suggest that the EphA2-RhoG signaling pathway is necessary but not sufficient for HGF-induced formation of extensions in MDCK cysts.

Our observations indicate that EphA2 is phosphorylated on S897 and promotes the formation of extensions in MDCK cysts downstream of HGF in a ligand-independent manner. By contrast, stimulation with the ligand ephrinA1 negates the effects of HGF on the formation of extensions in MDCK cysts, consistent with a previous report (Miao et al., 2003). Our results also demonstrate that stimulation with ephrinA1 results in the dephosphorylation of EphA2 on S897. These results suggest that EphA2 contributes to the remodeling of epithelia during development through liganddependent and -independent mechanisms, and that the ligand ephrinA1 functions as a negative regulator of EphA2 ligand- 
A

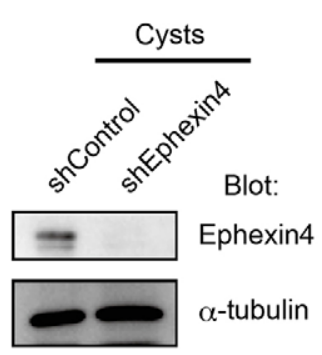

B
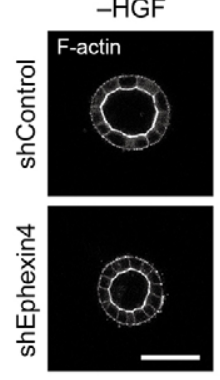
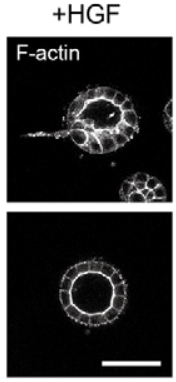
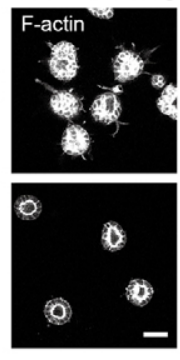

C

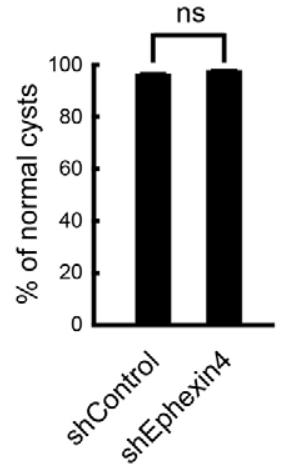

D

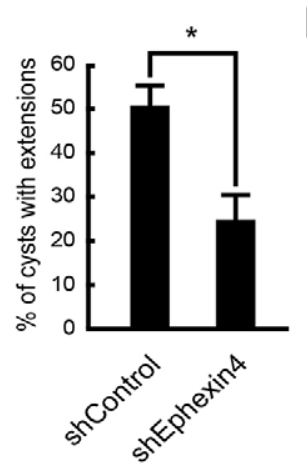

E

\section{Ephexin4-WT}

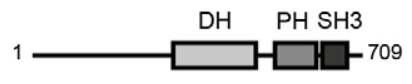

Ephexin4-AA

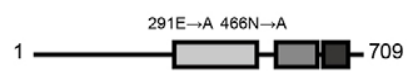

G

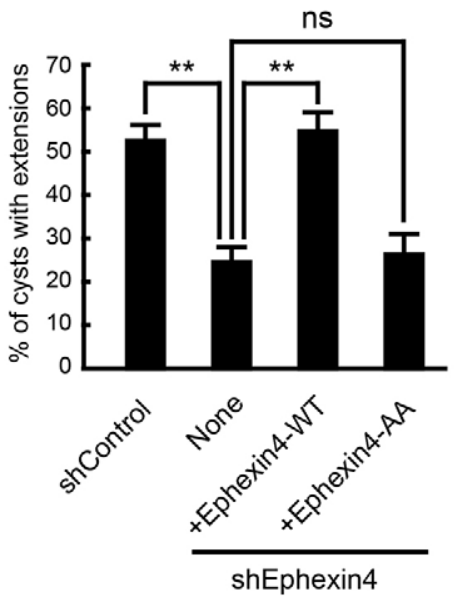

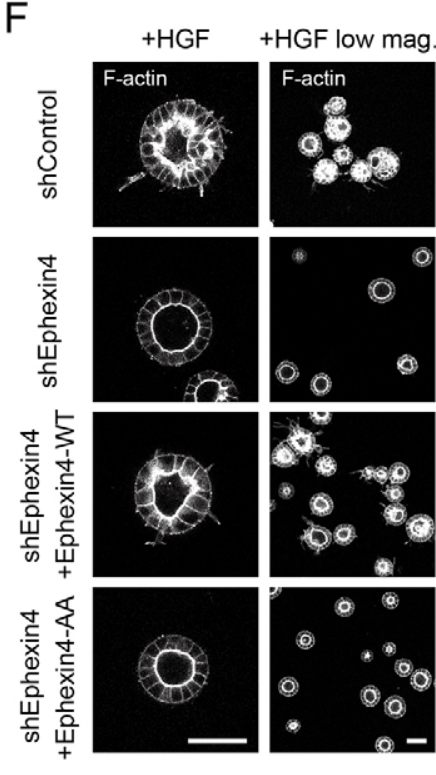

Fig. 5. Ephexin4 mediates HGF-induced formation of extensions through the activation of RhoG.

(A) Lysates from MDCK cysts expressing shControl or the shRNA against ephexin4 (shEphexin4) that had been grown for 10 days were analyzed by immunoblotting with antibodies against ephexin4 and $\alpha$-tubulin. (B) MDCK cysts expressing shControl or shEphexin4 were cultured in the presence or absence of HGF. Then they were stained with phalloidin. (C) Quantification of the percentage of normal cysts Data are the means \pm s.e.m. of three independent experiments (ns, not significant; Student's t-test). (D Quantification of cysts with one or more actin-rich extensions. Data are the means \pm s.e.m. of three independent experiments ( ${ }^{*} P<0.05$; Student's $t$-test). At least 100 cysts were counted for each experiment. (E) The ephexin4 constructs used in this study. $\mathrm{DH}$, Dbl homology domain; $\mathrm{PH}$, pleckstrin homology domain; SH3, Src homology domain 3. Numbers indicate amino acid position within the sequence. (F) MDCK cells expressing the indicated shRNAs or co-expressing the Flag-tagged ephexin4 constructs (-WT or -AA) were treated with HGF for 48 hours and stained with phalloidin. (G) Quantification of cysts with one or more actin-rich extensions. Data are the means \pm s.e.m. of five independent experiments $\left({ }^{* *} P<0.01\right.$; ns, not significant; one-way ANOVA with Dunnett T3), and at least 100 cysts were counted for each experiment. Low mag., low magnification. Scale bars: $50 \mu \mathrm{m}$. independent signaling. Miao et al. have also demonstrated that stimulation with ephrinA1 causes the retraction of preexisting cell protrusions that had been induced by HGF in MDCK cells cultured in collagen gel (Miao et al., 2003). In this study, we stimulated MDCK cysts with HGF and ephrinA1 for the same period of time (after 24 hours of treatment with HGF, cells were stimulated with ephrinA1 and cultured for an additional 24 hours), and found that stimulation with ephrinA1 caused dephosphorylation of EphA2 at S897 and suppressed the formation of extensions that were induced by HGF in our culture model. Therefore, it is possible that dephosphorylation of EphA2 at S897 is sufficient to retract preexisting extensions in MDCK cysts. We would like to use an inducible EphA2-S897A mutant or EphA2 knockdown to address this issue in future studies.

RhoG functions as a key upstream regulator of Rac1 (Katoh et al., 2000; Hiramoto-Yamaki et al., 2010). In MDCK cells, treatment with HGF stimulates Rac1 activity and induces phosphorylation of a downstream Rac1 effector, PAK, both of which are suppressed upon stimulation with ephrinA1 (Miao et al., 2003). In addition, inactivation of Rac1 inhibits HGF-induced formation of extensions (Miao et al., 2003; Yagi et al., 2012b). Therefore, RhoG might contribute to extension formation in HGF-stimulated MDCK cysts by regulating Rac1 activity at the basal membrane, downstream of EphA2 and ephexin4. By contrast, inactivation of RhoA or its downstream effector ROCK enhances HGF-induced formation of extensions and attenuates the inhibitory effect of ephrinA1 (Miao et al., 2003; Yu et al., 2003). Further studies are needed to elucidate whether phosphorylation of EphA2 at S897 mediates the formation of extensions in MDCK cysts by regulating Rac1 and/or RhoA activity.

EphA2 is overexpressed in a variety of human cancers (Pasquale, 2010; Boyd et al., 2014), and a correlation between the level of 
A

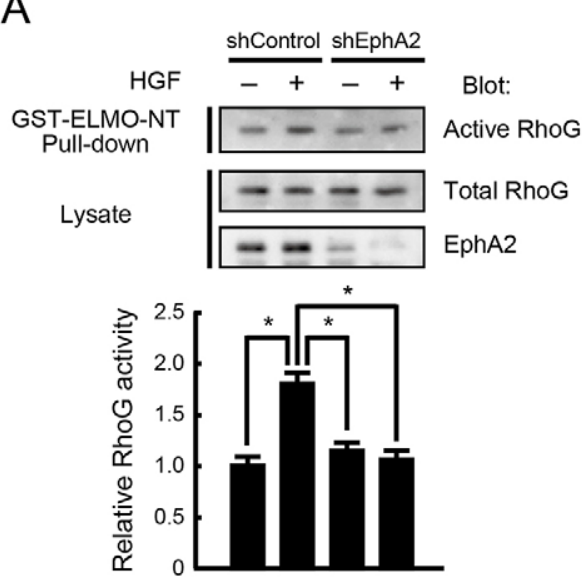

C
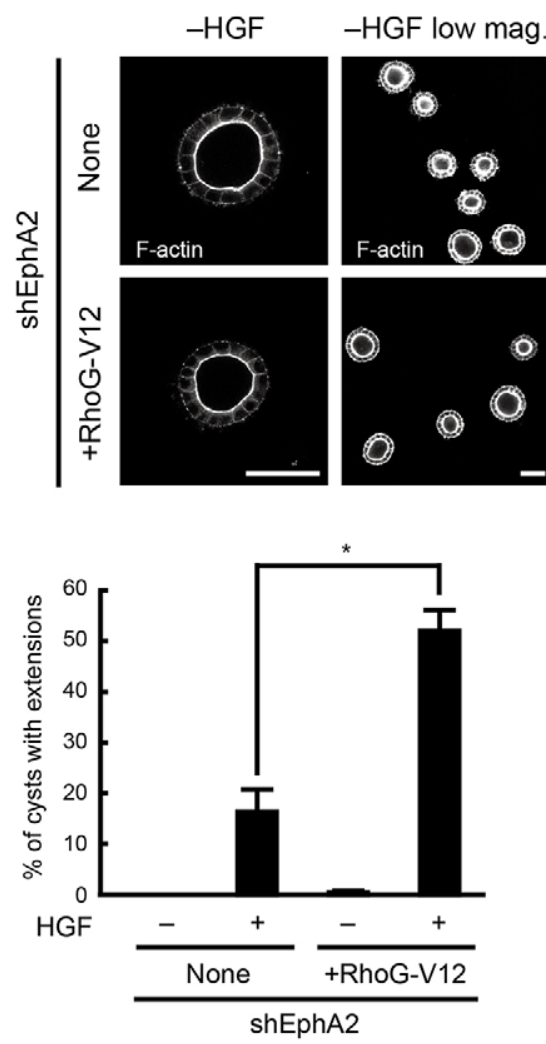

phosphorylation of EphA2 at S897 and malignant progression has been reported (Miao et al., 2009). Thus, these findings suggest that the spatial and temporal regulation of phosphorylation at S897 could be important for the formation and maintenance of epithelial tissues.

\section{MATERIALS AND METHODS}

\section{Plasmids and antibodies}

The expression plasmids encoding human EphA2 and Flag-tagged human ephexin4 were obtained, as described previously (Hiramoto-Yamaki et al., 2010), and subcloned into pcDNA3 vector (Life Technologies). EphA2SA (S897A) was constructed as described previously (Kawai et al., 2013). EphA2-KM (K646M) and Ephexin4-AA (E291A/N466A) were constructed by using PCR-mediated mutagenesis and subcloned into pcDNA3 vector. Myc-tagged human RhoG was generated as described previously (Katoh et al., 2000). EphA2 and Ephexin4 shRNAs were designed to target 21 nucleotides of the canine EphA2 transcript (5'GGAGGGTGTCGTCTCCAAATA-3') and the canine ephexin4 transcript
Fig. 6. EphA2 mediates HGF-induced RhoG activation. $(A, B) M D C K$ cells expressing the indicated plasmids were treated with $50 \mathrm{ng} / \mathrm{ml}$ HGF for 1 minute at room temperature. Cell lysates were incubated with GST-ELMO-NT, and bound endogenous RhoG and total cell lysates were analyzed using antibodies against RhoG and EphA2. Relative RhoG activity was determined by the amount of RhoG bound to GST-ELMO-NT normalized to the amount of RhoG in cell lysates, as analyzed by using Image J software. Data are presented as the means \pm s.e.m. from three or four independent experiments $\left({ }^{*} P<0.05\right.$; one-way ANOVA with Dunnett T3). (C) MDCK cells expressing shEphA2 or co-expressing GFP-tagged RhoGV12 were treated with HGF for 48 hours and stained using phalloidin. Low mag., low magnification. (D) Quantification of cysts with one or more actin-rich extensions. Data were the means \pm s.e.m. of three independent experiments $\left({ }^{*} P<0.05 ;\right.$ ns, not significant; one-way ANOVA with Dunnett T3), and at least 100 cysts were counted for each experiment. Scale bars: $50 \mu \mathrm{m}$.
(5'-GCTACCTATTTCTGTTCAATG-3'). The targets of each transcript were designed with no substantial identity to any mammalian gene sequence and were expressed by using an shRNA expression vector, pSilencer-hygro (Life Technologies). Control shRNA was designed to target 19 nucleotides of luciferase, as described previously (Harada et al., 2011).

The following antibodies were used in this study: a mouse monoclonal antibody against Myc, a rabbit polyclonal antibody against EphA2 (C-20), and a rat monoclonal antibody against RhoG (1F3 B3 E5) (Santa Cruz Biotechnology); mouse monoclonal antibodies against Flag (M2) and $\alpha-$ tubulin (Sigma); a rabbit polyclonal antibody against phosphorylated EphA2 (at residue S897) (Cell Applications); rabbit monoclonal antibodies against phosphorylated EphA2 (at residue S897; clone D9A1) and phosphorylated EphA2 (at residue Y588; clone D7X2L, Cell Signaling); a mouse polyclonal antibody against EphA2 (D7, Millipore); secondary antibodies conjugated to horseradish peroxidase (DAKO); and secondary antibodies conjugated to Alexa Fluor 488 (Invitrogen). F-actin was visualized with Rhodamine-phalloidin 
(Invitrogen). The antibody against ephexin4 has been described previously (Hiramoto-Yamaki et al., 2010).

\section{Cell culture and transfection}

MDCK cells were a gift from Etsuko Kiyokawa (Kanazawa Medical University, Ishikawa, Japan). HEK293T and MDCK cells were grown in Dulbecco's modified Eagle's medium containing 10\% fetal bovine serum, $4 \mathrm{mM}$ glutamine, 100 units $/ \mathrm{ml}$ of penicillin and $0.1 \mathrm{mg} / \mathrm{ml}$ of streptomycin under humidified air containing $5 \% \mathrm{CO}_{2}$ at $37^{\circ} \mathrm{C}$. Cells were transfected with indicated expression vectors using Lipofectamine Plus (for HEK293T cells) or Lipofectamine 2000 (for MDCK cells) (Life Technologies) according to the manufacturer's instructions. In some experiments, MDCK cells were used after selection with $400 \mu \mathrm{g} / \mathrm{ml}$ of hygromycin-B or $500 \mu \mathrm{g} /$ $\mathrm{ml}$ of $\mathrm{G} 418$

\section{Formation of cysts and extensions}

Cyst and cyst extension formation were performed as previously described (Pollack et al., 1998; Martin-Belmonte et al., 2007; Yagi et al., 2012a). To form cysts, MDCK cells were plated at a density of $7.5 \times 10^{3}$ cells onto 13-mm-diameter coverslips coated with $20 \mu \mathrm{l}$ of growth-factor-reduced Matrigel (BD Biosciences) in 24-well plates and cultured in medium containing $2 \%$ growth-factor-reduced Matrigel for 10 days. For cyst extension formation, MDCK cysts were grown in Matrigel for 8 days and treated for 48 hours with $50 \mathrm{ng} / \mathrm{ml}$ HGF (R\&D Systems) in medium containing 2\% growth-factor-reduced Matrigel. In some experiments, cysts were treated with $1 \mu \mathrm{g} / \mathrm{ml}$ control-Fc or ephrinA1-Fc (R\&D Systems) for 24 hours. The percentage of cysts with extensions was calculated by counting the number of cysts with one or more actin-rich extensions protruding from their basal surface, according to the quantification method described previously (Eastburn et al., 2012).

\section{Immunoblotting}

To detect the level of phosphorylation of EphA2 at S897 in MDCK cysts, cysts on Matrigel were incubated in $1.25 \mathrm{mM}$ EDTA in PBS on ice for 1 hour to depolymerize Matrigel and then centrifuged for 5 minutes at $1500 \mathrm{~g}$. After cysts had been washed twice with PBS, proteins were eluted in Laemmli sample buffer. Proteins were separated by using SDS-PAGE, and were transferred onto a polyvinylidene difluoride membrane (Millipore Corporation) using electrophoresis. The membrane was blocked with 3\% low fat milk in Tris-buffered saline and then incubated with primary antibodies. The primary antibodies were detected with horseradish-peroxidase-conjugated secondary antibodies and enhanced chemiluminescence (ECL) detection kit (ECL Prime, GE Healthcare; Chemi-Lumi one, Nacalai Tesque). To examine the involvement of the PI3K-Akt pathway, cysts were treated with $20 \mu \mathrm{M}$ LY294002 (a PI3Kspecific inhibitor, Calbiochem) or $1 \mu \mathrm{M}$ MK-2206 (an Akt-specific inhibitor, ChemieTek).

\section{Measurement of RhoG activity}

Measurement of RhoG activity in cells was performed as described previously (Katoh and Negishi, 2003). The N-terminal RhoG-binding domain of ELMO2 (ELMO-NT, amino acids 1-362) was expressed in Escherichia coli as a fusion protein with GST and purified on glutathioneSepharose beads. The purified protein was suspended with $10 \mathrm{mM}$ Tris$\mathrm{HCl}, \mathrm{pH} 7.5,150 \mathrm{mM} \mathrm{NaCl}, 2 \mathrm{mM} \mathrm{MgCl} \mathrm{m}_{2}$ and $0.1 \mathrm{mM}$ DTT and stored at $-80^{\circ} \mathrm{C}$. Protein concentration was determined by comparing with BSA standards after SDS-PAGE and by staining with Coomassie Brilliant Blue. To determine RhoG activity in MDCK cells, cells were serum-starved and then treated with $50 \mathrm{ng} / \mathrm{ml} \mathrm{HGF}$ for 1 minute at room temperature. The cells were lysed with the ice-cold cell lysis buffer $(10 \mathrm{mM}$ Tris-HCl, $\mathrm{pH} 7.5$, $100 \mathrm{mM} \mathrm{NaCl}, 2 \mathrm{mM} \mathrm{MgCl} 2,1 \%$ Nonidet P-40, $10 \%$ glycerol, $1 \mathrm{mM} \mathrm{DTT}$, $1 \mathrm{mM}$ PMSF, $10 \mu \mathrm{g} / \mathrm{ml}$ Aprotinin and $10 \mu \mathrm{g} / \mathrm{ml}$ Leupeptin) containing $20 \mu \mathrm{g}$ of GST-ELMO-NT. Cell lysates were then centrifuged for 5 minutes at $10,000 \mathrm{~g}$ at $4^{\circ} \mathrm{C}$, and the supernatants were incubated with glutathioneSepharose beads for 30 minutes at $4^{\circ} \mathrm{C}$. The beads were washed with the lysis buffer, and bound proteins were analyzed by using SDS-PAGE and immunoblotting.

\section{Immunofluorescence microscopy}

Cysts on Matrigel-coated coverslips were fixed with 4\% paraformaldehyde in PBS for 30 minutes at room temperature and washed twice with PBS. After residual paraformaldehyde had been quenched with $50 \mathrm{mM} \mathrm{NH}_{4} \mathrm{Cl}$ in PBS, cysts were permeabilized with $0.5 \%$ Triton X-100 in PBS for 10 minutes and incubated with $10 \%$ goat serum in PBS for 30 minutes to block nonspecific antibody binding. Then cysts were incubated with primary antibodies in Can Get Signal Immunostain Immunoreaction Enhancer Solution A (TOYOBO) overnight at $4^{\circ} \mathrm{C}$. After washing with PBS, cells were incubated with Rhodamine-phalloidin and a secondary antibody conjugated with Alexa Fluor 488 for 2 hours, washed with PBS for 30 minutes and mounted upside down on glass-bottomed dishes in $90 \%$ glycerol containing $0.1 \%$ p-phenylenediamine dihydrochloride in PBS. To visualize cyst structure, cysts were incubated with $10 \%$ FBS in PBS for 30 minutes and incubated with Rhodamine-phalloidin in blocking buffer for 2 hours. Images were acquired using a laser-scanning confocal imaging system (EZ-C1 version 3.20 software; Nikon) and a microscope (Eclipse TE2000-U; Nikon) with a $60 \times /$ NA 1.40 oil objective (Nikon) and a digital camera (DXM1200C; Nikon). All images were prepared using Photoshop (Adobe).

\section{Data analysis}

Statistical significance was established using the two-sample Student's $t$-test or the analysis of variance (ANOVA) and post-hoc test (Dunnett T3) (SPSS software version 16.0, IBM). $P<0.05$ was considered significant.

\section{Acknowledgements}

We thank Dr Etsuko Kiyokawa (Kanazawa Medical University, Ishikawa, Japan) for her technical advice on three-dimensional cell culture in Matrigel and for providing MDCK cells

\section{Competing interests}

The authors declare no competing or financial interests.

\section{Author contributions}

K.H. and H.K. designed and performed the experiments, analyzed the data, discussed results and wrote the paper; M.N. analyzed the data and discussed results

\section{Funding}

This work was supported in part by Grants-in-aid for Scientific Research from the Ministry of Education, Science, Sports, and Culture of Japan [Scientific Research (B) 23370085] and by a grant from Suzuken Memorial Foundation.

\section{Supplementary material}

Supplementary material available online at

http://jcs.biologists.org/lookup/suppl/doi:10.1242/jcs.163790/-/DC1

\section{References}

Akada, M., Harada, K., Negishi, M. and Katoh, H. (2014). EphB6 promotes anoikis by modulating EphA2 signaling. Cell. Signal. 26, 2879-2884.

Batson, J., Maccarthy-Morrogh, L., Archer, A., Tanton, H. and Nobes, C. D. (2014). EphA receptors regulate prostate cancer cell dissemination through Vav2RhoA mediated cell-cell repulsion. Biol. Open 3, 453-462.

Boissier, P., Chen, J. and Huynh-Do, U. (2013). EphA2 signaling following endocytosis: role of Tiam1. Traffic 14, 1255-1271.

Boyd, A. W., Bartlett, P. F. and Lackmann, M. (2014). Therapeutic targeting of EPH receptors and their ligands. Nat. Rev. Drug Discov. 13, 39-62.

Brantley-Sieders, D. M., Caughron, J., Hicks, D., Pozzi, A., Ruiz, J. C. and Chen, J. (2004). EphA2 receptor tyrosine kinase regulates endothelial cell migration and vascular assembly through phosphoinositide 3-kinase-mediated Rac1 GTPase activation. J. Cell Sci. 117, 2037-2049.

Cowan, C. W., Shao, Y. R., Sahin, M., Shamah, S. M., Lin, M. Z., Greer, P. L., Gao, S., Griffith, E. C., Brugge, J. S. and Greenberg, M. E. (2005). Vav family GEFs link activated Ephs to endocytosis and axon guidance. Neuron 46, 205-217.

Eastburn, D. J., Zegers, M. M. and Mostov, K. E. (2012). Scrib regulates HGFmediated epithelial morphogenesis and is stabilized by Sgt1-HGP90. J. Cell Sci. 125, 4147-4157.

Egea, J. and Klein, R. (2007). Bidirectional Eph-ephrin signaling during axon guidance. Trends Cell Biol. 17, 230-238.

Ellerbroek, S. M., Wennerberg, K., Arthur, W. T., Dunty, J. M., Bowman, D. R., DeMali, K. A., Der, C. and Burridge, K. (2004). SGEF, a RhoG guanine nucleotide exchange factor that stimulates macropinocytosis. Mol. Biol. Cell 15, 3309-3319. 
Fang, W. B., Brantley-Sieders, D. M., Parker, M. A., Reith, A. D. and Chen, J. (2005). A kinase-dependent role for EphA2 receptor in promoting tumor growth and metastasis. Oncogene 24, 7859-7868.

Fang, W. B., Ireton, R. C., Zhuang, G., Takahashi, T., Reynolds, A. and Chen, J. (2008a). Overexpression of EPHA2 receptor destabilizes adherens junctions via a RhoA-dependent mechanism. J. Cell Sci. 121, 358-368.

Fang, W. B., Brantley-Sieders, D. M., Hwang, Y., Ham, A. J. and Chen, J. (2008b). Identification and functional analysis of phosphorylated tyrosine residues within EphA2 receptor tyrosine kinase. J. Biol. Chem. 283, 16017-16026.

Harada, K., Hiramoto-Yamaki, N., Negishi, M. and Katoh, H. (2011). Ephexin4 and EphA2 mediate resistance to anoikis through RhoG and phosphatidylinosito 3-kinase. Exp. Cell Res. 317, 1701-1713.

Hiramoto, K., Negishi, M. and Katoh, H. (2006). Dock4 is regulated by RhoG and promotes Rac-dependent cell migration. Exp. Cell Res. 312, 4205-4216.

Hiramoto-Yamaki, N., Takeuchi, S., Ueda, S., Harada, K., Fujimoto, S., Negishi, M. and Katoh, H. (2010). Ephexin4 and EphA2 mediate cell migration through a RhoG-dependent mechanism. J. Cell Biol. 190, 461-477.

Hunter, S. G., Zhuang, G., Brantley-Sieders, D., Swat, W., Cowan, C. W. and Chen, J. (2006). Essential role of Vav family guanine nucleotide exchange factors in EphA receptor-mediated angiogenesis. Mol. Cell. Biol. 26, 4830-4842.

Irie, F. and Yamaguchi, Y. (2002). EphB receptors regulate dendritic spine development via intersectin, Cdc42 and N-WASP. Nat. Neurosci. 5, 1117-1118.

Katoh, H., Yasui, H., Yamaguchi, Y., Aoki, J., Fujita, H., Mori, K. and Negishi, M. (2000). Small GTPase RhoG is a key regulator for neurite outgrowth in PC12 cells. Mol. Cell. Biol. 20, 7378-7387.

Katoh, H. and Negishi, M. (2003). RhoG activates Rac1 by direct interaction with the Dock180-binding protein Elmo. Nature 424, 461-464.

Katoh, H., Hiramoto, K. and Negishi, M. (2006). Activation of Rac1 by RhoG regulates cell migration. J. Cell Sci. 119, 56-65.

Kawai, H., Kobayashi, M., Hiramoto-Yamaki, N., Harada, K., Negishi, M. and Katoh, H. (2013). Ephexin4-mediated promotion of cell migration and anoikis resistance is regulated by serine 897 phosphorylation of EphA2. FEBS Open Bio 3, 78-82.

Kullander, K. and Klein, R. (2002). Mechanisms and functions of Eph and ephrin signalling. Nat. Rev. Mol. Cell Biol. 3, 475-486.

Lin, K. T., Gong, J., Li, C. F., Jang, T. H., Chen, W. L., Chen, H. J. and Wang, L. H (2012). Vav3-rac1 signaling regulates prostate cancer metastasis with elevated Vav3 expression correlating with prostate cancer progression and posttreatment recurrence. Cancer Res. 72, 3000-3009.

Lindberg, R. A. and Hunter, T. (1990). cDNA cloning and characterization of eck an epithelial cell receptor protein-tyrosine kinase in the eph/elk family of protein kinases. Mol. Cell. Biol. 10, 6316-6324.

Margolis, S. S., Salogiannis, J., Lipton, D. M., Mandel-Brehm, C., Wills, Z. P., Mardinly, A. R., Hu, L., Greer, P. L., Bikoff, J. B., Ho, H. Y. H. et al. (2010). EphBmediated degradation of the RhoA GEF Ephexin5 relieves a developmental brake on excitatory synapse formation. Cell 143, 442-455.

Martin-Belmonte, F., Gassama, A., Datta, A., Yu, W., Rescher, U., Gerke, V. and Mostov, K. (2007). PTEN-mediated apical segregation of phosphoinositides controls epithelial morphogenesis through Cdc42. Cell 128, 383-397.

Miao, H., Nickel, C. H., Cantley, L. G., Bruggeman, L. A., Bennardo, L. N. and Wang, B. (2003). EphA kinase activation regulates HGF-induced epithelia branching morphogenesis. J. Cell Biol. 162, 1281-1292.

Miao, H., Li, D. Q., Mukherjee, A., Guo, H., Petty, A., Cutter, J., Basilion, J. P. Sedor, J., Wu, J., Danielpour, D. et al. (2009). EphA2 mediates liganddependent inhibition and ligand-independent promotion of cell migration and invasion via a reciprocal regulatory loop with Akt. Cancer Cell 16, 9-20.

Miao, H., Gale, N. W., Guo, H., Qian, J., Petty, A., Kaspar, J., Murphy, A. J., Valenzuela, D. M., Yancopoulos, G., Hambardzumyan, D. et al. (2015). EphA2 promotes infiltrative invasion of glioma stem cells in vivo through cross-talk with Akt and regulates stem cell properties. Oncogene 34, 558-567.
O'Brien, L. E., Zegers, M. M. and Mostov, K. E. (2002). Building epithelial architecture: insights from three-dimensional culture models. Nat. Rev. Mol. Cell Biol. 3, 531-537.

Parri, M., Buricchi, F., Giannoni, E., Grimaldi, G., Mello, T., Raugei, G., Ramponi, G. and Chiarugi, P. (2007). EphrinA1 activates a Src/focal adhesion kinase-mediated motility response leading to rho-dependent actino/myosin contractility. J. Biol. Chem. 282, 19619-19628.

Pasquale, E. B. (2010). Eph receptors and ephrins in cancer: bidirectional signalling and beyond. Nat. Rev. Cancer 10, 165-180.

Penzes, P., Beeser, A., Chernoff, J., Schiller, M. R., Eipper, B. A., Mains, R. E. and Huganir, R. L. (2003). Rapid induction of dendritic spine morphogenesis by trans-synaptic ephrinB-EphB receptor activation of the Rho-GEF kalirin. Neuron 37, 263-274.

Pitulescu, M. E. and Adams, R. H. (2010). Eph/ephrin molecules - a hub for signaling and endocytosis. Genes Dev. 24, 2480-2492.

Pollack, A. L., Runyan, R. B. and Mostov, K. E. (1998). Morphogenetic mechanisms of epithelial tubulogenesis: MDCK cell polarity is transiently rearranged without loss of cell-cell contact during scatter factor/hepatocyte growth factor-induced tubulogenesis. Dev. Biol. 204, 64-79.

Sahin, M., Greer, P. L., Lin, M. Z., Poucher, H., Eberhart, J., Schmidt, S., Wright T. M., Shamah, S. M., O’Connell, S., Cowan, C. W. et al. (2005). Eph-dependent tyrosine phosphorylation of ephexin1 modulates growth cone collapse. Neuron 46, 191-204.

Shamah, S. M., Lin, M. Z., Goldberg, J. L., Estrach, S., Sahin, M., Hu, L, Bazalakova, M., Neve, R. L., Corfas, G., Debant, A. et al. (2001). EphA receptors regulate growth cone dynamics through the novel guanine nucleotide exchange factor ephexin. Cell 105, 233-244

Tolias, K. F., Bikoff, J. B., Kane, C. G., Tolias, C. S., Hu, L. and Greenberg, M. E. (2007). The Rac1 guanine nucleotide exchange factor Tiam1 mediates EphB receptor-dependent dendritic spine development. Proc. Natl. Acad. Sci. USA 104 7265-7270.

Vaught, D., Chen, J. and Brantley-Sieders, D. M. (2009). Regulation of mammary gland branching morphogenesis by EphA2 receptor tyrosine kinase. Mol. Biol. Cell 20, 2572-2581.

Wakayama, Y., Miura, K., Sabe, H. and Mochizuki, N. (2011). EphrinA1-EphA2 signal induces compaction and polarization of Madin-Darby canine kidney cells by inactivating Ezrin through negative regulation of RhoA. J. Biol. Chem. 286, 44243-44253

Wang, Y., Ota, S., Kataoka, H., Kanamori, M., Li, Z., Band, H., Tanaka, M. and Sugimura, H. (2002). Negative regulation of EphA2 receptor by Cbl. Biochem. Biophys. Res. Commun. 296, 214-220.

Yagi, S., Matsuda, M. and Kiyokawa, E. (2012a). Suppression of Rac1 activity at the apical membrane of MDCK cells is essential for cyst structure maintenance. EMBO Rep. 13, 237-243.

Yagi, S., Matsuda, M. and Kiyokawa, E. (2012b). Chimaerin suppresses Rac1 activation at the apical membrane to maintain the cyst structure. PLOS ONE 7 e52258

Yamaki, N., Negishi, M. and Katoh, H. (2007). RhoG regulates anoikis through a phosphatidylinositol 3-kinase-dependent mechanism. Exp. Cell Res. 313 2821-2832.

Yu, W., O’Brien, L. E., Wang, F., Bourne, H., Mostov, K. E. and Zegers, M. M. (2003). Hepatocyte growth factor switches orientation of polarity and mode of movement during morphogenesis of multicellular epithelial structures. Mol. Biol. Cell 14, 748-763.

Zegers, M. M., O’Brien, L. E., Yu, W., Datta, A. and Mostov, K. E. (2003). Epithelial polarity and tubulogenesis in vitro. Trends Cell Biol. 13, 169-176.

Zhuang, G., Hunter, S., Hwang, Y. and Chen, J. (2007). Regulation of EphA2 receptor endocytosis by SHIP2 lipid phosphatase via phosphatidylinositol 3 Kinase-dependent Rac1 activation. J. Biol. Chem. 282, 2683-2694. 


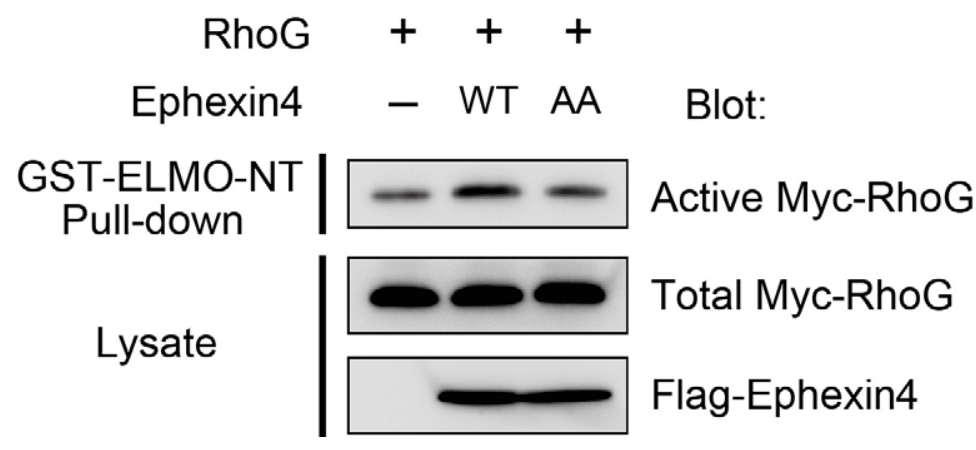

Fig. S1. Effect of Ephxein4-WT or Ephexin4-AA expression on RhoG activity. HEK293T cells were co-transfected with Myc-tagged RhoG and Flag-tagged Ephexin4 (-WT or -AA). Cell lysates were incubated with GST-ELMO-NT, and bound Myc-tagged RhoG and total cell lysates were analyzed with antibodies against RhoG and Flag. 